\title{
Search for Supersymmetry at the LHC
}

\author{
Christian Autermann ${ }^{1, a}$ \\ on behalf of the ATLAS and CMS collaborations \\ ${ }^{1}$ I. Phys. Inst. B, RWTH Aachen University, Germany
}

\begin{abstract}
Searches for supersymmetry at the ATLAS and CMS experiments are discussed. The analyzed data were recorded at the Large Hadron Collider in 2015 at a center of mass energy of $13 \mathrm{TeV}$ and correspond to an integrated luminosity of up to $3 \mathrm{fb}^{-1}$ per experiment. No physics beyond the standard model has been observed. Different inclusive and specialized analysis strategies targeting various signal scenarios are introduced. The analysis sensitivities are summarized as exclusion contours for different simplified signal model spectra and compared to the previous $8 \mathrm{TeV}$ results.
\end{abstract}

\section{Introduction}

After the long shutdown, the Large Hadron Collider (LHC) at CERN has delivered in 2015 the first proton-proton collision data at a center of mass energy of $13 \mathrm{TeV}$. The two experiments, ATLAS [1] and CMS [2], have currently analyzed the data corresponding to an integrated luminosity of up to $3 \mathrm{fb}^{-1}$. The collaborations focus strongly on searches for physics beyond the standard model, especially after the discovery of the Higgs boson [3, 4]. The sensitivity to signals of new physics scenarios already matches or exceeds the results obtained on the $8 \mathrm{TeV}$ data set collected until 2012 corresponding to up to $20 \mathrm{fb}^{-1}$.

Supersymmetry (SUSY) [5] is a very popular theory for physics beyond the standard model, developed since the 1970s as an extension of the Poincaré symmetry. SUSY links each particle of the standard model (SM) with a supersymmetric partner with equal properties, except for the spin which differs by $1 / 2$ and the mass. The supersymmetry fixes the hierarchy problem associated with the divergent first-loop order correction terms to the Higgs-mass parameter $m_{H}^{2}$. SUSY provides a dark matter candidate particle if the lightest supersymmetric particle (LSP) is neutral and stable, e.g. due to $R$-parity conservation. The unification of gauge couplings at a high energy scale becomes possible in supersymmetry, motivating believes in the existence of grand unified theories.

The standard model of particle physics is very successfully describing the fundamental particles and their interactions studied at the collider experiments. Excellent agreement between theory predictions and measurements at the ATLAS and the CMS experiments is observed over all studied production and decay channels. A particular example is the observation of the $B_{s}^{0} \rightarrow \mu^{+} \mu^{-}$decay and the measurement of its branching fraction [6]. This decay is rare in the SM because it is suppressed at loop- or box-diagram level to an order, at which also new particles such as predicted from supersymmetry may contribute and interfere. The measured branching fractions are consistent with standard

\footnotetext{
a e-mail: auterman@cern.ch
} 


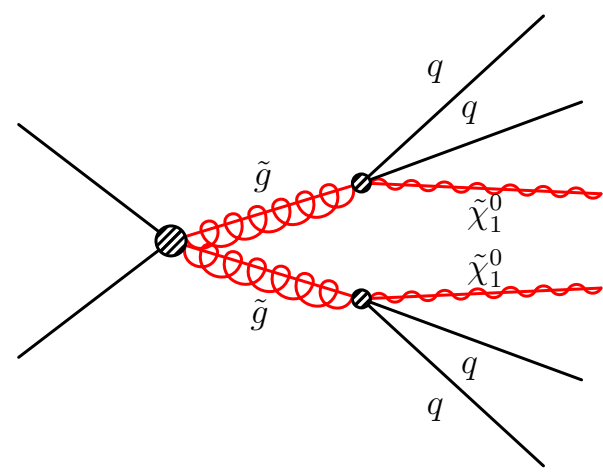

(a) $\tilde{g} \tilde{g}$, decay via off-shell squarks

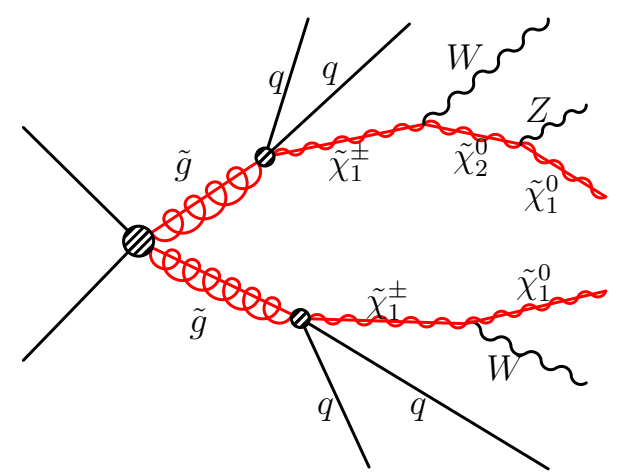

(b) $\tilde{g} \tilde{g}$, decay via off-shell squarks and heavy gauginos

Figure 1. Effective Feynman diagrams for strong production of gluinos leading to highly energetic jets (a) or to final states with large jet multiplicities (b), depending on the gluino and neutralino masses.

model expectations and can be used to constrain models of supersymmetry [7]. In order to interpret the analyis results generally simplified model spectra (SMS) $[8,9]$ are used, which allow to model individual decay topologies through effective on-shell theories, involving only the masses of typically two SUSY particles as free parameters.

The good understanding of SM physics requires dedicated searches for new physics. Different strategies for searches for supersymmetry are discussed, from very inclusive searches for strong production in hadronic final states to more specific signatures such as dilepton resonances and kinematic edges are discussed in the following.

\section{Direct inclusive searches for strong production of SUSY}

Strong production of supersymmetry and decay channels into fully hadronic final states, as shown in Fig. 1, typically have the largest production cross sections and branching fractions and offer therefore generally good signal sensitivities. As SUSY is a broken symmetry, the SUSY partner masses in the supermultiplets differ. Supersymmetric particle masses are typically heavy, due to existing direct limits and SM precision measurements. Events containing SUSY particles contain therefore more energy than the SM background and accumulate in the high energy tails of distributions like $E_{T}^{\text {miss }}$. Various other signal sensitive variables such as $M_{T 2}$ [10], $\alpha_{T}$ [11], or "Razor" $[12,13]$ are used to enrich the signal selection. The classic variable missing transverse energy $E_{T}^{\text {miss }}$, utilized e.g. in $[14,15]$, is defined as the absolute value of the vectorial sum of all reconstructed particle momenta, and is therefore sensitive to the amount of energy of particles that did not interact with the detector, such as neutrinos or the supersymmetry LSPs.

The precise estimation of the standard model background contributions to the tails of the signal sensitive variables is of crucial importance to achieve sensitive results. In the case of the fully hadronic final states this SM background is due to QCD multijet production, where the artificial $E_{T}^{\text {miss }}$ is caused mostly by jet energy resolution effects, and by $t \bar{t}, W+$ jets, and $Z \rightarrow v \bar{v}$ processes with real $E_{T}^{\text {miss }}$ from hard neutrinos and no reconstructed leptons. Especially the QCD multijet processes are hard to simulate with Monte Carlo methods, as on one hand the various effects leading to $E_{T}^{\text {miss }}$ are very rare and hard to model and on the other hand the background production cross section is very large requiring large statistics. 


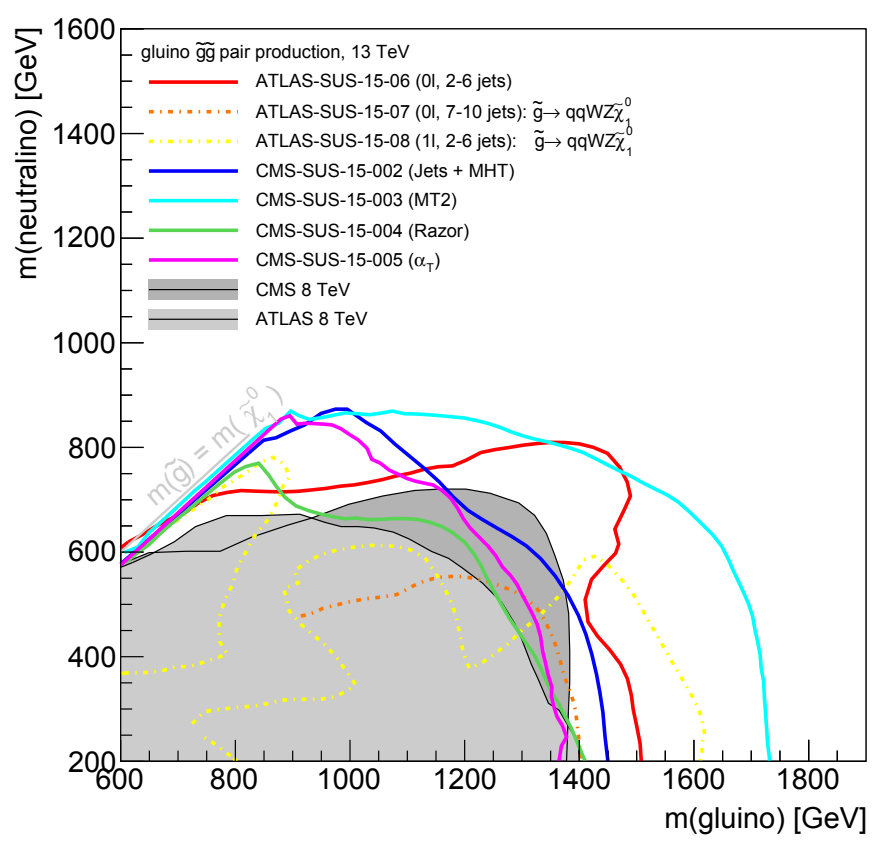

Figure 2. Resulting exclusion contours for inclusive searches in the fully-hadronic final state for simplified scenarios of gluino-pair production with either short decays via off-shell squarks $\tilde{g} \rightarrow q q \tilde{\chi}_{1}^{0}$ into highly energetic jets or longer decays $\tilde{g} \rightarrow q q Z \tilde{\chi}_{1}^{0}$ leading to higher jet multiplicities. ATLAS exclusion contours are taken from $[14,16,17]$, CMS from taken from [10-12, 15], the gray shaded areas correspond to the envelope of all $8 \mathrm{TeV}$ contours, taken from [18-20].

The estimation of the standard model background is often improved by the use of data sideband regions, where either the Monte Carlo simulation is corrected and validated, or events kinematically similar to the background in the signal region are extracted. The discussed results from the same experiment, the same signal scenario, and therefore a similar final state are not necessarily strongly correlated, because of the set of different signal-sensitive variables and the rich variety of data-driven background estimation strategies.

In Fig. 2 the exclusion contours with respect to the gluino and the lightest neutralino masses for gluino-pair production scenarios are shown for the different inclusive supersymmetry search analyses performed at ATLAS with data corresponding to an integrated luminosity of $3.2 \mathrm{fb}^{-1}$ and at CMS with $2.2 \mathrm{fb}^{-1}$ of data. The best sensitivity of the analyses performed on data collected at a center-ofmass energy of $8 \mathrm{TeV}$ and about $20 \mathrm{fb}^{-1}$ of data are shown as a gray area [20]. Two $\tilde{g} \tilde{g}$ SMS with different gluino decays leading to different final state particle topologies are shown. Results for long cascades, where the gluinos decay through charginos into the neutralino LSP lead to many jets and $W / Z$-bosons in the final state and are shown dashed. High jet multiplicities are targeted by [16], while the $W / Z$ may lead to leptons which are analyzed by [17,21]. Direct gluino decays $\tilde{g} \rightarrow q q \tilde{\chi}_{1}^{0}$ through squarks assumed to be off-shell lead to rather low multiplicities of energetic jets, significant $E_{T}^{\text {miss }}$ due to the two neutralinos $\tilde{\chi}_{1}^{0}$ LSPs, and no leptons. This challenging final state requires analyses 


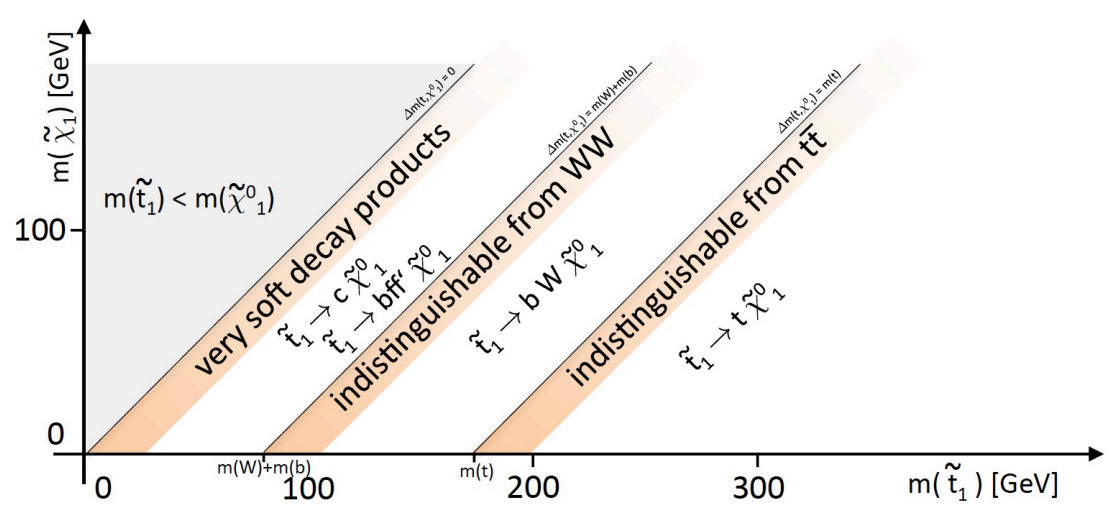

Figure 3. Phase-space region of the stop decay for $\tilde{t}_{1} \tilde{t}_{1}$ pair production in the $\mathrm{m}\left(\tilde{t}_{1}\right)$ and $\mathrm{m}\left(\tilde{\chi}_{1}^{0}\right)$ plane [20]. The decay topology and the kinematics of the decay products differ significantly, requiring a rich set of experimental analyses.

with sophisticated background estimation strategies [10-15]. While CMS typically uses multiple exclusive signal region combined into one likelihood for best signal sensitivity, ATLAS typically uses one dedicated single-channel region per signal scenario to allow for easier reinterpretations of the result. Further interpretations in more simplified scenarios and cross-section limits can be found under the quoted references.

\section{Third generation squark searches}

Naturalness arguments motivate searches for direct production of third generation squarks, i.e. top and bottom squarks. The third generation has strong couplings to the Higgs sector, therefore third generation squarks are required to remain light, at the order of the $\mathrm{TeV}$ scale, in order to not reintroduce a new little hierarchy problem. Pair production of top squarks may lead to different decay channels depending on the mass difference to the lightest neutralino $\Delta m=\mathrm{m}(\tilde{t})-\mathrm{m}\left(\tilde{\chi}_{1}^{0}\right)$ as illustrated in Fig. 3. Different analysis strategies are developed, to cover the stop and neutralino mass space with optimal sensitivity.

In the case of large mass differences $\Delta m$ between the stop and the neutralino on-shell top-quarks are produced in the $\tilde{t_{1}} \rightarrow t \tilde{\chi}_{1}^{0}$ decay, as shown in Fig. 4. The SM top transverse momentum $p_{T}$ depends on $\Delta m$, so that the signal can be distinguished from SM top-pair production. For smaller $\Delta m$ on-shell top quarks or on-shell $W$-bosons may no longer occur in the stop decays $\tilde{t}_{1} \rightarrow b f f^{\prime} \tilde{\chi}_{1}^{0}$, leading to final states with only a small amount of visible energy. If the mass gap $\Delta m$ is even smaller than the $b$-quark mass, only the flavor changing decay $\tilde{t}_{1} \rightarrow c \tilde{\chi}_{1}^{0}$ into a $c$-jet of very low energy and $E_{T}^{\text {miss }}$ is possible. In particular if the mass gap $\Delta m$ is equal to the top-mass or to the $W$-boson mass it becomes very difficult to distinguish the potential $\tilde{t}_{1} \tilde{t}_{1}$ signal from standard model top-pair production or from SM $W$-pair production. Here, dedicated analyses using e.g. the spin-spin correlation or the SM top-pair cross-section are developed.

In the first $3 \mathrm{fb}^{-1}$ of the $13 \mathrm{TeV}$ data several searches, comparable to the inclusive analyses discussed before but using $b$-tagged jet information, have been performed in the all-hadronic final state [22, 23], and in the final state with one lepton [24, 25], or with two leptons [26]. These analyses 


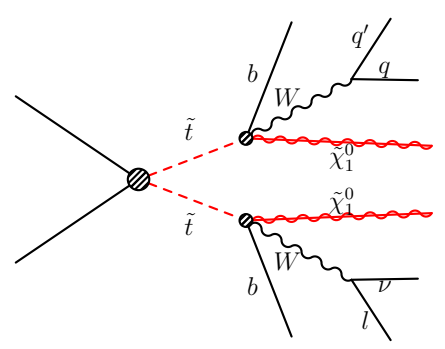

(a) $\tilde{t} \tilde{t} \rightarrow b b W W \tilde{\chi}_{1}^{0} \tilde{\chi}_{1}^{0}$

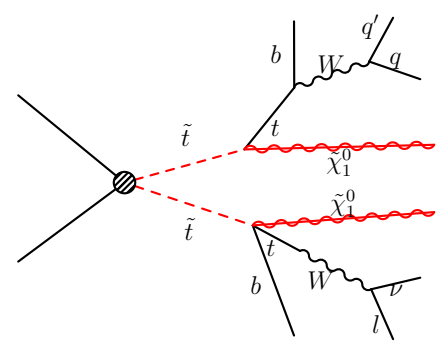

(b) $\tilde{t} \tilde{t} \rightarrow t t \tilde{\chi}_{1}^{0} \tilde{\chi}_{1}^{0}$

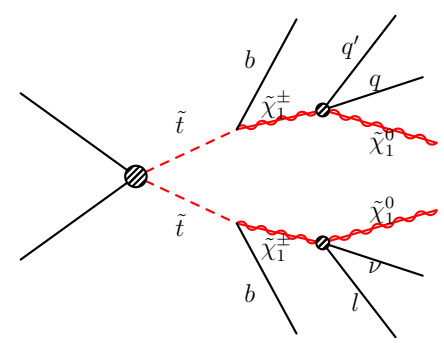

(c) $\tilde{t} \tilde{t} \rightarrow b \bar{b} \tilde{\chi}_{1}^{ \pm} \tilde{\chi}_{1}^{ \pm}$

Figure 4. Effective Feynman diagrams for direct stop production and the following stop three body decay into the neutralino, a $b$-quark and an on-shell $W$-boson (a), an on-shell top (b), or through trough a chargino $\tilde{t} \rightarrow b \tilde{\chi}_{1}^{ \pm}$ followed by a three-body decay of the $\tilde{\chi}_{1}^{ \pm}(\mathrm{c})$.

probe the phase-space where the standard model particles from the stop decay have significant energy and can be clearly separated from SM processes, as illustrated in Fig. 5. The lightest stop mass up to $800 \mathrm{GeV}$ depending on $\mathrm{m}\left(\tilde{\chi}_{1}^{0}\right)$ can be excluded for simplified $\tilde{t}_{1} \tilde{t}_{1}$-production scenarios.

At low $\Delta m$, the decay products of the off-shell top-quarks and $W$-bosons have only low energy and are hard to identify. Analyses [27] of final states with at least one soft lepton but large missing transverse momentum can probe top squark masses below $340 \mathrm{GeV}$ for small mass gaps of $\Delta m=$ $50 \mathrm{GeV}$. Mono-jet analyses [28] provide additional sensitivity. Here, the most energetic jet is most likely due to initial-state radiation, while the visible standard model particles of the stop quark decay do not pass the reconstruction thresholds. Previous analyses carried out on the $8 \mathrm{TeV}$ data [20] shown as gray area in Fig. 5 made also use of $c$-tagging algorithms, that attempt to identify the low energetic $c$-jet of the flavor changing stop decay possible at low $\Delta m$.

\section{Searches for gauge-mediated broken supersymmetry}

In models of gauge-mediated supersymmetry breaking the lightest SUSY particle is the gravitino $\tilde{G}$, the spin-3/2 partner of the spin-2 graviton. The experimental signature of these models is determined by the nature of the next-to-lightest SUSY particle (NLSP). The results discussed in the following assume that the NLSP is the lightest neutralino $\tilde{\chi}_{1}^{0}$. The neutralino decays $\tilde{\chi}_{1}^{0} \rightarrow B \tilde{G}$ into the gravitino LSP and a neutral standard model boson $B$, which can be either $\gamma, Z^{0}$, or $H$ as illustrated in Fig. 6 . The branching fraction depends on the neutralino mass and mixing, the $\tilde{\chi}_{1}^{0}$ lifetime depends on the gravitino mass. The $\mathrm{m}(\tilde{G})$ and thus the $\tilde{\chi}_{1}^{0}$ lifetime are assumed to be negligible.

Bino- and wino-like neutralino mixings may lead to up to two photons in the final state. The analyses [29, 30] carried out on the first $3 \mathrm{fb}^{-1}$ of $13 \mathrm{TeV}$ data already exceed the sensitivity of previous analyses [20] as shown in Fig. 7. Final states with photons arise in the standard model from QCD multijet like $\gamma+$ jet production, initial-, and final state radiation of photons. Additionally, misidentified jets or electrons may be reconstructed as photons. The "fake photon" background from misidentified electrons can be modeled using a sample of isolated well identified electrons, that pass all signal selection cuts, except for the photon identification. This sample is then weighted according to the electron identification $\epsilon_{e}$ efficiency and the electron misidentification probability $f_{e \rightarrow \gamma}$. Because $f_{e \rightarrow \gamma}$ is about 50 times smaller than $\epsilon_{e}$, the statistical precision of this data-driven approach is very good. 


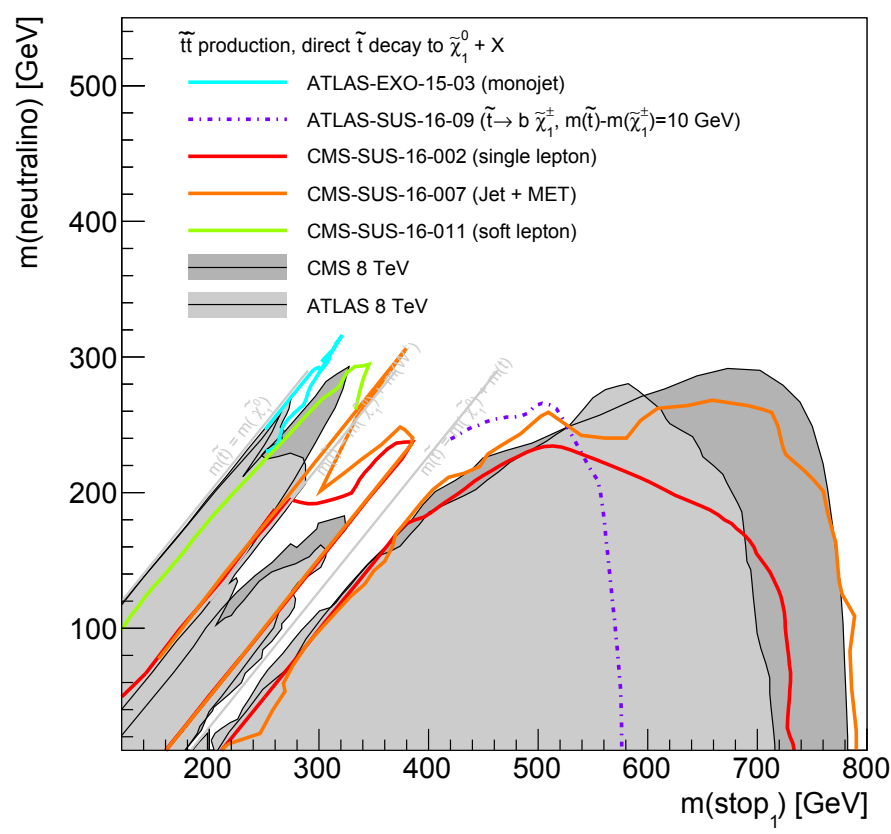

Figure 5. Selected ATLAS [28] and CMS [23, 25, 27] exclusion contours for top squark pair production $\tilde{t}_{1} \tilde{1}_{1}$ directly decaying via on- or off-shell top-quarks and $W$-bosons $\tilde{t}_{1} \rightarrow b f f^{\prime} \tilde{\chi}_{1}^{0}$ or results [26] for decays via charginos $\tilde{t}_{1} \rightarrow b \tilde{\chi}_{1}^{ \pm} \rightarrow b f f^{\prime} \tilde{\chi}_{1}^{0}$. The $8 \mathrm{TeV}$ contour envelopes are from [18-20].

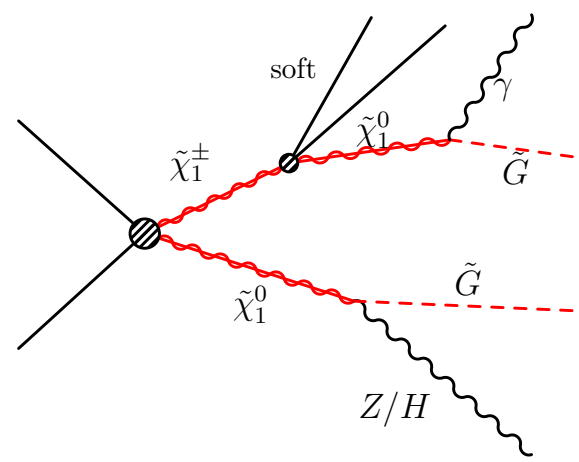

(a) $\tilde{g} \tilde{g}$, decay via off-shell squarks

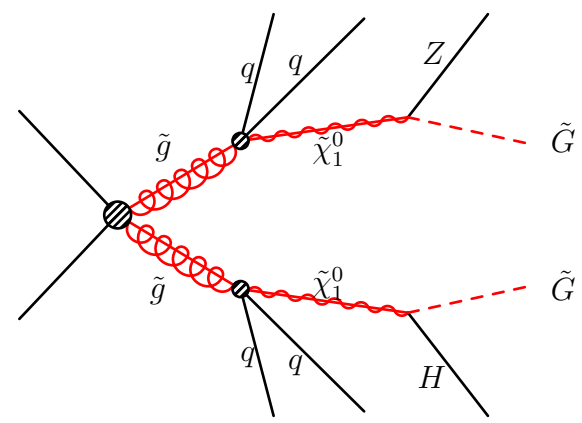

(b) $\tilde{g} \tilde{g}$, decay via off-shell squarks and heavy gauginos

Figure 6. Effective Feynman diagrams for SUSY models with gauge-mediated symmetry breaking for electroweak production (a) and strong production (b). The type of the standard model boson created together with the gravitino depends generally on the mixing of the neutralino mother particle. 


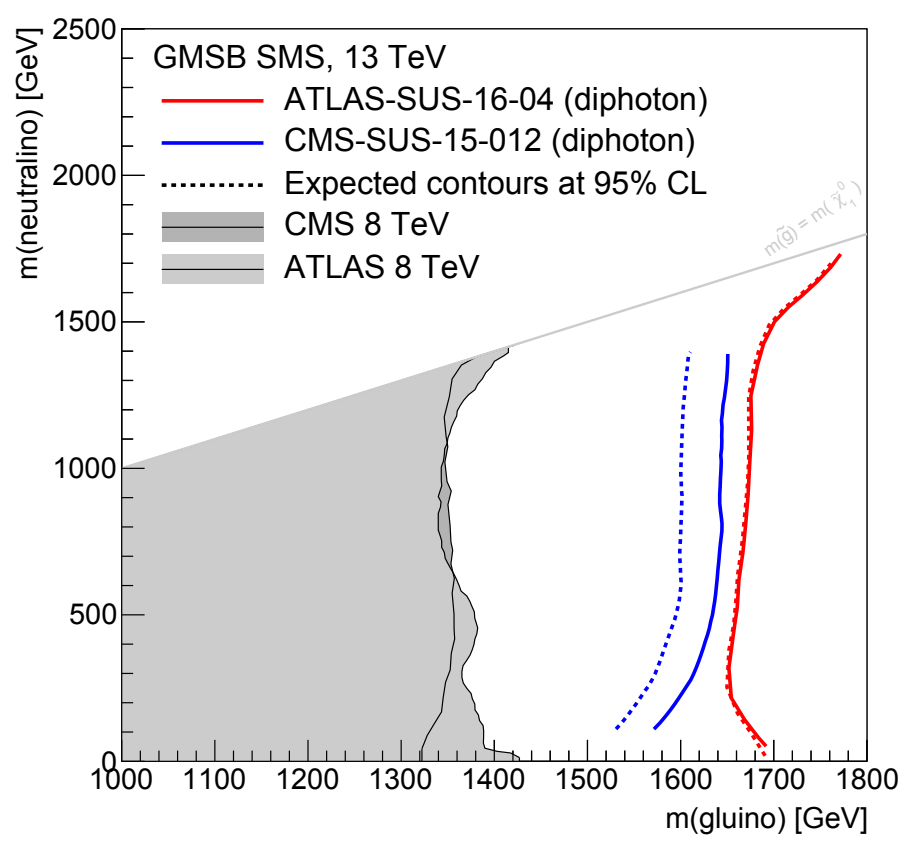

Figure 7. Resulting exclusion contours at $13 \mathrm{TeV}[29,30]$ compared to the results at $8 \mathrm{TeV}[31,32]$ for gluinopair production $\tilde{g} \tilde{g}$ within gauge-mediated symmetry breaking SUSY scenarios with bino-like neutralinos and $\tilde{g} \rightarrow q q \tilde{\chi}_{1}^{0} \rightarrow q q \gamma \tilde{G}$ leading to final states with diphotons, jets, and $E_{T}^{\text {miss }}$.

\section{Dilepton mass-edge and resonance searches}

Supersymmetry searches for dilepton resonances or mass-edges are typical examples for highly sensitive and optimized analyses for specific signal scenarios. The signal in the dilepton invariant mass $m_{l l}$ spectrum has the form of a sharp peak or a triangular shape. Heavy neutral SUSY particles can decay into a light neutral particle such as the neutralino LSP via a slepton $\tilde{\chi}_{2}^{0} \rightarrow l \tilde{l} \rightarrow l l \tilde{\chi}_{1}^{0}$ or a standard model boson $\tilde{\chi}_{2}^{0} \rightarrow Z \tilde{\chi}_{1}^{0} \rightarrow l l \tilde{\chi}_{1}^{0}$ as shown in Fig. 9(a). The slepton can be off- or on-shell, which influences slightly the curvature of the lower edge of the triangular shaped signal in the invariant dilepton mass spectrum. The position of the upper edge is determined by the mass difference of the two involved neutral SUSY particles $\Delta m=\mathrm{m}\left(\tilde{\chi}_{2}^{0}\right)-\mathrm{m}\left(\tilde{\chi}_{1}^{0}\right)$.

The prominent dilepton resonance or edge-like shape signal is an important feature of the analysis, but the excellent signal sensitivity is also due to the very reliable and precise estimation of the standard model backgrounds. The dilepton final state with $E_{T}^{\text {miss }}$ and jets is dominated to a large fraction by standard model $t t$-production and similar backgrounds symmetric in lepton flavor. Lepton flavor-symmetric (SF) backgrounds contain as many $e^{+} e^{-}+\mu^{+} \mu^{-}$events as there are $\mu^{+} e^{-}+\mu^{-} e^{+}$ events, while the signal does not contain opposite-flavor (OF) lepton events. The amount of flavor symmetric backgrounds in the SF signal region can therefore be estimated [33] from a OF control region. Differences in electron and muon efficiencies lead to small corrections that can be derived by explicitly measuring the relevant electron and muon efficiencies or by measuring the $R_{\mathrm{SF} / \mathrm{FF}}$ difference in data sidebands. The systematic uncertainty of this method, i.e. the uncertainty on $R_{\mathrm{SF} / \mathrm{OF}}$ is about $4 \%$ 

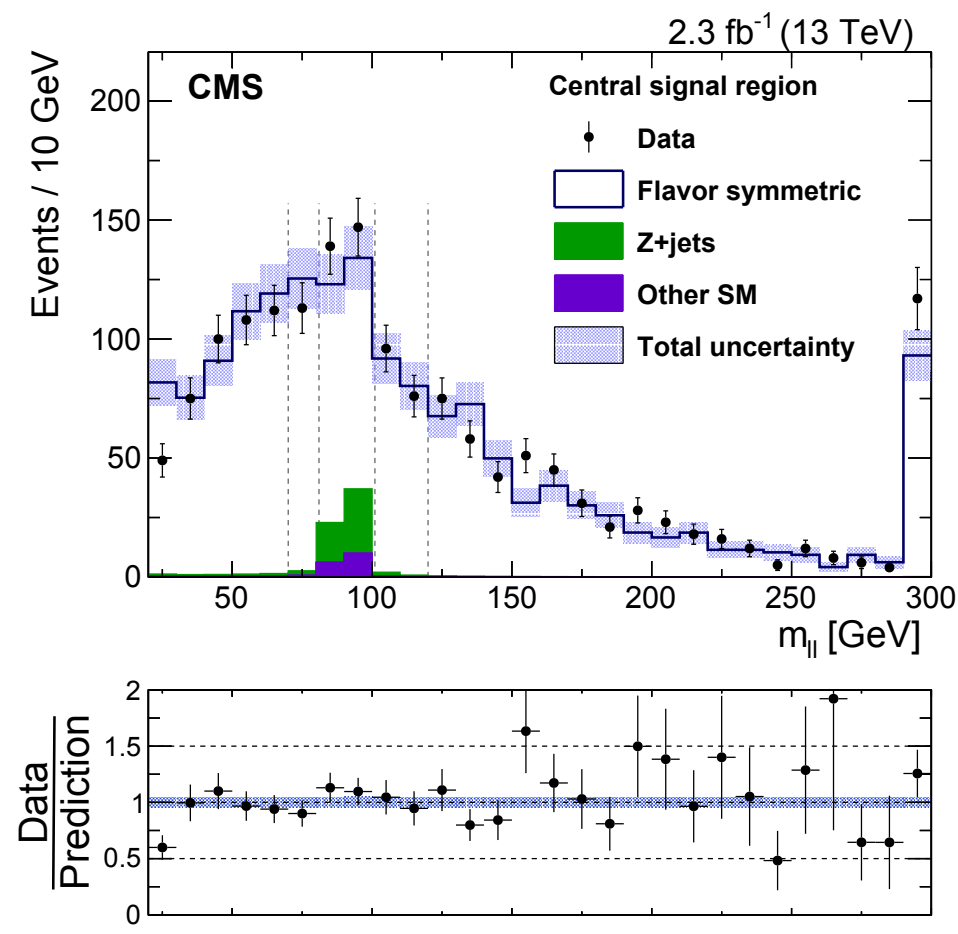

Figure 8. Invariant dimuon mass distribution for the data compared to the standard model background estimation [33] in the region, where the Run-I analysis [35] reported an excess using $20 \mathrm{fb}^{-1}$ of $8 \mathrm{TeV}$ data.

and of the same order as the statistical uncertainty of the OF control region, which is of the order of the irreducible statistical uncertainty of the data.

The precise determination of the subdominant $Z / \gamma^{*} \rightarrow l^{+} l^{-}$background is relevant in particular for signal resonances on the $Z$-peak. Here [34], the similarity between $\gamma$-jet events used as data control sample with $Z+$ jets events is exploited. In both processes the $E_{T}^{\text {miss }}$ originates from similar mechanisms such as jet resolution and mismeasurements and not from genuine unobserved particles. The control sample is selected with the same kinematic cuts as applied on the data, and the events are reweighed to match the boson $p_{T}$ distribution of the $Z \gamma^{*}$ events. Differences in resolution between photons and muons are taken into account. The $E_{T}^{\text {miss }}$ of the events in the control sample is recalculated and is normalized to the data in a sideband region.

The analyses carried out in the $8 \mathrm{TeV}$ data $[35,36]$ received some attention, because excesses of the order of $2.5-3.0 \sigma$ were observed in the low invariant dilepton mass region at CMS, and on the Z-resonance at ATLAS, respectively. The ATLAS and CMS analyses have been repeated on the first set of data recorded at $13 \mathrm{TeV}$. ATLAS [34] has analyzed $3.2 \mathrm{fb}^{-1}$ of data and finds good agreement of the data and background expectation in invariant dilepton mass regions below and above the $Z$-region, not confirming the excess observed by CMS in $8 \mathrm{TeV}$, but again finds an excess on the $Z$-peak corresponding to $2.2 \sigma$. CMS [33] has analyzed $2.2 \mathrm{fb}^{-1}$ of $13 \mathrm{TeV}$ data finding generally good agreement between data and standard model expectations as shown in Fig. 8, ruling out the possibility 


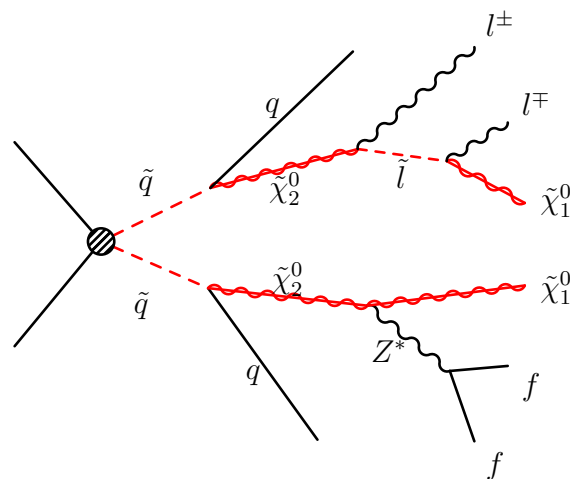

(a)

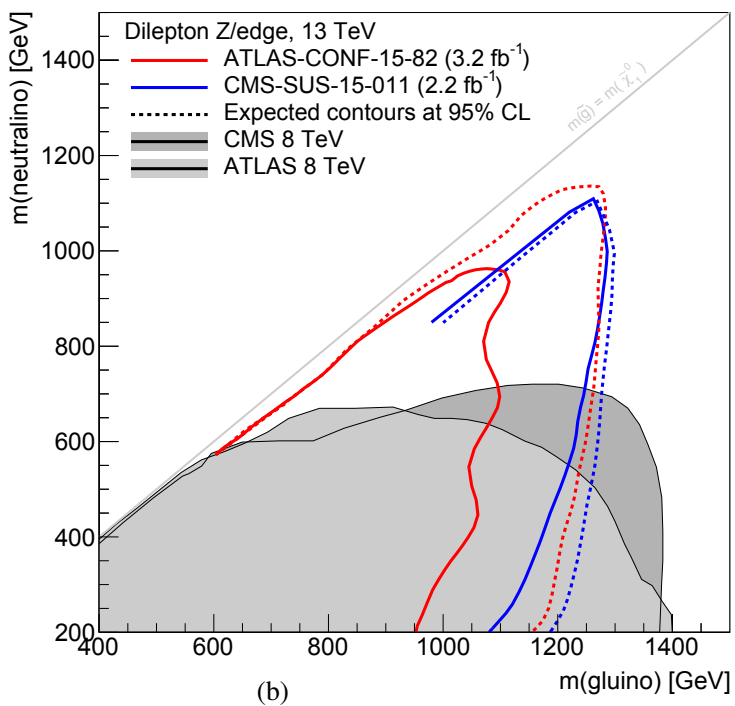

(b)

Figure 9. Effective Feynman diagram for sbottom pair production leading to a final state with dileptons (a). In the upper cascade a heavy neutralino $\tilde{\chi}_{2}^{0}$ decays via a slepton $\tilde{l}$ into a light neutralino $\tilde{\chi}_{1}^{0}$, the invariant mass of the dilepton system exhibits a characteristic triangular shaped form. In the lower cascade dileptons through the $Z$-resonance can be produced. The exclusion contours obtained at $13 \mathrm{TeV}[33,34]$ for a simplified scenario of gluino pair-production $\tilde{g} \tilde{g}$ with dilepton resonant decays $\tilde{g} \rightarrow q q Z \tilde{\chi}_{1}^{0}$ and negligible neutralino LSP masses $\mathrm{m}\left(\tilde{\chi}_{1}^{0}\right)=1 \mathrm{GeV}$ is compared to the $8 \mathrm{TeV}$ contours $[35,36]$ in (b).

that the $8 \mathrm{TeV}$ excesses were due to a SUSY signal. The resulting exclusion contours for a simplified scenario of gluino pair-production leading to resonant decays through Z-bosons are shown in Fig. 9(b) comparing the $8 \mathrm{TeV}$ and $13 \mathrm{TeV}$ results of ATLAS and CMS.

\section{Conclusion}

The ATLAS and CMS collaborations have searched in the first $3 \mathrm{fb}^{-1}$ of data collected at $13 \mathrm{TeV}$ for signs of supersymmetry. The observed data are in good agreement with the expectations from standard model processes. Limits on supersymmetric model parameters and masses in simplified model spectra have been derived that begin to exceed previous results obtained with data collected at lower center-of-mass energies. A wide range of signal scenarios, production and decay channels, and SUSY particle mass regions have been studied using sophisticated analysis methods in particular for the data-driven estimation of the SM backgrounds. The LHC continued to deliver about $40 \mathrm{fb}^{-1}$ of $13 \mathrm{TeV}$ data in 2016, that will be analyzed for the upcoming conferences. Natural supersymmetry is expected at the $\mathrm{TeV}$ scale, the data currently being collected will soon allow to challenge these scenarios.

\section{References}

[1] ATLAS Collaboration, JINST 3, S08003 (2008) 
[2] CMS Collaboration, JINST 3, S08004 (2008)

[3] ATLAS Collaboration, Phys. Lett. B 716, 1 (2013), hep-ex/1207.7214

[4] CMS Collaboration, Phys. Lett. B 716, 30 (2012), hep-ex/1207.7235

[5] S.P. Martin, Adv. Ser. Direct. High Energy Phys. 18 (1997), hep-ph/9709. 356

[6] LHCb, CMS Collaborations, Nature 522, 68 (2015), hep-ex/1411.4413

[7] P. Bechtle et al. (2015), hep-ph/1508.05951

[8] N. Arkani-Hamed, P. Schuster, N. Toro, J. Thaler, L.T. Wang et al. (2007), hep-ph/0703.088

[9] J. Alwall, P. Schuster, N. Toro, Phys. Rev. D 79, 075020 (2009), hep-ph/0810. 3921

[10] CMS Collaboration, JHEP 10, 006 (2016), hep-ex/1603. 04053

[11] CMS Collaboration, Submitted to Eur. Phys. J. C (2016), hep-ex/1611. 00338

[12] CMS Collaboration, Submitted to Phys. Rev. D (2016), hep-ex/1609.07658

[13] ATLAS Collaboration, Eur. Phys. J. C73, 2362 (2013), hep-ex/1212.6149

[14] ATLAS Collaboration, Eur. Phys. J. C76, 392 (2016), hep-ex/1605.03814

[15] CMS Collaboration, Phys. Lett. B758, 152 (2016), hep-ex/1602 . 06581

[16] ATLAS Collaboration, Phys. Lett. B757, 334 (2016), hep-ex/1602 .06194

[17] ATLAS Collaboration, Eur. Phys. J. C76, 565 (2016), hep-ex/1605.04285

[18] ATLAS Collaboration, Public Results (2016), https://twiki.cern.ch/twiki/bin/view/AtlasPublic/SupersymmetryPublicResults

[19] CMS Collaboration, Public Results (2016), http://cms-results. web.cern.ch/cms-results/public-results/publications/

[20] C. Autermann, Prog. Part. Nucl. Phys. 90, 125 (2016), hep-ex/1609.01686

[21] CMS Collaboration, subm. to Phys. Rev. D (2016), hep-ex/1609. 09386

[22] CMS Collaboration, PAS SUS-16-001 (2016), https://cds.cern.ch/record/2140975

[23] CMS Collaboration, PAS SUS-16-007 (2016), https://cds.cern.ch/record/2141543

[24] ATLAS Collaboration, Phys. Rev. D94, 052009 (2016), 1606.03903

[25] CMS Collaboration, PAS SUS-16-002 (2016), https://cds.cern.ch/record/2139650

[26] ATLAS Collaboration, CONF-2016-009 (2016), https://cds.cern.ch/record/2139643

[27] CMS Collaboration, PAS SUS-16-011 (2016), https://cds.cern.ch/record/2161097

[28] ATLAS Collaboration, Phys. Rev. D94, 032005 (2016), hep-ex/1604.07773

[29] ATLAS Collaboration, Eur. Phys. J. C76, 517 (2016), 1606.09150

[30] CMS Collaboration, PAS SUS-15-012 (2016), https://cds.cern.ch/record/2143897

[31] ATLAS Collaboration, Phys. Rev. D92, 072001 (2015), 1507.05493

[32] CMS Collaboration, Phys. Rev. D92, 072006 (2015), 1507.02898

[33] CMS Collaboration, Accepted by JHEP (2016), hep-ex/1607.00915

[34] ATLAS Collaboration, CONF-2015-082 (2015), https://cds.cern.ch/record/2114854

[35] CMS Collaboration, JHEP 04, 124 (2015), 1502.06031

[36] ATLAS Collaboration, Eur. Phys. J. C75, 318 (2015), [Erratum: Eur. Phys. J.C75,no.10,463(2015)], hep-ex/1503.03290 